of $Q$ there is, for any given temperature, a definite heat flux at which the line changes slope by up to $25 \%$. The effect is at its largest near $2 \mathrm{~K}$, gradually diminishing as the temperature is reduced.

It is now more than thirty years since the existence of the thermal boundary resistance was discovered by Kapitza (J. Phys. U.S.S.R., 4, 181; 1941) and it seems at first sight very surprising that in the course of the large body of experimental work on the phenomenon since that time, no one has reported observation of the anomalous features now being described. The effect would clearly be easily missed, however, except in cases where a large number of closely spaced data points were taken at small values of $Q$. Ironically, there is perhaps some evidence for the existence of these anomalies in the original graphs published by Kapitza.

As for the origin of the anomalies, the authors discuss a number of possibilities the most plausible of which concerns the onset of turbulence in the helium very close to the metal surface. Liquid helium at these temperatures, $\mathrm{He}$ II, can be regarded as an intimate mixture of two interpenetrating but extremely dissimilar fluids: a normal fluid component which behaves much like any other liquid and which carries all the thermal energy of the helium and a superfluid component which carries no thermal energy, has zero viscosity and is thus responsible for many of the more bizarre properties of the liquid. When heat enters the liquid, a counterflow of the two components occurs, with superfluid approaching the heated metal, being converted into normal fluid and then flowing away again. The authors tentatively conclude that the observed anomalous behaviour arises from the onset of turbulence in the normal fluid component within the very thin layer of helium at the surface of the metal, but further work will be necessary before this interpretation can be confirmed.

The authors have taken considerable pains to try and ensure that they have not been observing a mere artefact arising from their measuring equipment or experimental techniques. If the phenomena which they report are genuine, however, any theory of Kapitza resistance under these conditions will need to take them properly into account, so it seems highly desirable that the experiment be repeated in another laboratory as soon as possible.

\section{Protein folding made simple}

from Barry Robson

IT is hardly surprising that nobody has yet predicted how a given protein molecule folds up, considering that there are very much simpler molecules whose conformations defy theoretical analysis. Theoreticians with insatiable appetites for protein molecules, however, can restore at least a modicum of common sense by first examining protein systems in which prediction of the folding process is probably a simpler task.

Ptitsyn and Rashin (Dokl. Acad. Nauk SSSR, 213, 473; 1974 and Biophys. Chem., in the press) have made the first attempt to predict the folding and final overall conformation of a globular protein from its amino acid sequence and known secondary (locally organised) structure. The relatively simple protein system is in this case the myoglobin molecule which along with its haemoglobin relatives, is exceptional in being composed of $\alpha$ helix and very little else. Ptitsyn and Rashin justify starting with the known helical regions in terms of the popular model in which such regions are among the first features to appear during the folding up of the polypeptide chain, a model which is consistent with experimental evidence (Jardetzky et al., Cold Spring Harb. Symp. quant. Biol., 36, 257; 1971; Robson and Pain, Proc. Jerusalem Symp. quant. Chem. Biochem., 5, 279; 1973; Sachs et al., Proc. natn. Acad. Sci. U.S.A., 69, 3790; 1972). Furthermore, recent publications confirm that $x$-helical regions in proteins can be predicted with reasonable accuracy (see, for example, Chou and Fasman, Biochemistry, 13, 222; 1974).

The method of Ptitsyn and Rashin is to try and predict the folding pathway and resulting most stable configuration of stacked a helices on the basis of hydrophobic interactions between the helices. They find that the most stable configuration "coincides in a rough resolution" with the native conformation. It is surprising, however, that they obtain even such an approximate structure, and it is necessary to ask whether such an agreement could be fortuitous. Helices were treated simply as cylinders with a radius of $5 \AA$, and dispersion, electrostatic and hydrogen bonding forces between helices are neglected except to the extent that they are included in the parameters of the hydrophobic interactions. Furthermore, a possible contribution of the helix backbone to the hydrophobic interactions was not investigated. Finally, the study was apparently carried out manually and because of the still very large number of possible configurations it would be difficult to exclude an accidental subjective element from entering such calculations where the answer is known in advance.

It is therefore possible that in retrospect the simplifications of Ptitsyn and Rashin will be seen to be too simple, and their work is likely to be best remembered for the more critical reassessments of the problem which it will undoubtedly stimulate. Nevertheless, the concept of interactions between secondary structure features such as $x$ helices leading to the formation of single compact globules is a potent one and extends well beyond the simple case of myoglobin. The recent work of Rossman and Liljas (J. molec. Biol., 85, 177; 1974) confirms and quantifies the idea that sections of the backbone of more complicated proteins are bunched up locally to form subglobules within the overall globular structure of the molecules, so dividing many protein molecules into two or more distinct lobes. Within such subglobules, interactions between $x$-helical regions and other secondary structure features are probably very important.

As the first protein to have its conformation determined by X-ray crystallography, myoglobin once surprised biochemists with its unexpected complexity and the lack of any obvious relation between amino acid sequence and conformation. Now the pendulum is beginning to swing from a state of general pessimism to a state of optimism which is probably not unjustifiable.

\section{All excited in Lisbon}

from a Correspondent

THE opulent ambience of the Calouste Gulbenkian Foundation Centre in Lisbon on April 18-24 was the choice for an international conference on excited states of biological molecules under the sponsorship of the European Physical Society and European Photochemical Association, with J. B. Birks (University of Manchester) as papers secretary and editor.

As both Sir George Porter (Royal Institution, London) and G. Eisinger (Bell Laboratories, New Jersey) noted in their lectures, 1974 is about 25 years since the seminal Faraday Society Discussion of 1950, when M. Kasha discussed the phophorescence of organic molecules in terms of the Jablonski diagram and singlet--triplet conversion, and about 50 years since Perrin postulated singlet-singlet energy transfer to explain the depolarisation of fluorescence. Excited states in biological molecules were last discussed at an international meeting at Arden House, New York, in October 1969 (see Nature, 226, 113-118; 1970). In the light of that report, the programme of this year's meeting had inevitably a slight sense of déja $v u$, as the main themes have not changed greatly in 5 years.

Porter reviewed the methods avail- 